6th International Conference on Industrial Engineering and Industrial Management.

XVI Congreso de Ingeniería de Organización. Vigo, July 18-20, 2012

\title{
Strategic Capacity Planning in KIOs: A Classification Scheme
}

\author{
Martínez $C^{2}$, Lusa $\mathbf{A}^{3}$, Mas $\mathbf{M}^{4}$, de la Torre $\mathbf{R}^{3}$, Mateo $\mathbf{M}^{3}$
}

\begin{abstract}
English) This paper introduces the Strategic Capacity Planning problem in knowledge intensive organizations (KIOs) and proposes a classification scheme based on different characteristics such as the organization structure, the workforce characteristics, the capacity requirements, the capacity decisions or the evaluation criteria, among others. The classification, which gives rise to a high number of variants, is the first step towards a general solving methodology design and the developing of ad hoc solving procedures.
\end{abstract}

Keywords: Strategic Capacity Planning, Long Term Capacity Planning, Staff Planning, KIO/KIF, KIBS.

\footnotetext{
${ }^{1}$ Supported by the Spanish Ministry of Economy and Competitivity (project DPI2010-15614).

${ }^{2}$ Carme Martínez

Departamento de Organización de Empresas / Instituto de Organización y Control / ETS de Ingeniería Industrial de Barcelona. Universitat Politècnica de Catalunya. e-mail:

mcarme.martinez@upc.edu

${ }^{3}$ Amaia Lusa $(\bowtie)$

Departamento de Organización de Empresas / Instituto de Organización y Control / ETS de Ingeniería Industrial de Barcelona. Universitat Politècnica de Catalunya. Av. Diagonal 647, planta 11, 08028 Barcelona, Spain

e-mail: amaia.lusa@upc.edu

${ }^{4}$ Marta Mas / Rocío de la Torre / Manel Mateo

Departamento de Organización de Empresas / ETS de Ingeniería Industrial de Barcelona. Universitat Politècnica de Catalunya. e-mail: marta.mas-machuca@upc.edu /

maria.rocio.de.torre@upc.edu/manel.mateo@upc.edu
} 


\subsection{Introduction}

This paper introduces a classification scheme for strategic capacity planning problems in knowledge intensive organizations (KIOs), which is mainly related to determining the sizes, types and scheduling of capacity expansion and reduction of skilled people as the main limiting factor of their capacity.

Strategic capacity planning is extremely important for every company (Luss, 1982; Olhager et al., 2001; Geng and Jiang, 2009). For manufacturing industries and some types of services (such as telecommunications, transport, electricity distribution, water distribution, etc.) companies have to invest huge amounts of money in tangible assets with long payout times (Paraskevopuolos et al., 1991), and sometimes these decisions are irreversible or the assets invested have insignificant resale, scrap or salvage value (Berman et al., 1994). For this reason, most of the developed capacity planning tools are focused on organizations that have significant expansion costs. In our study we will focus in service organizations for which the main expansion cost is related to personal and the workforce planning constitutes a major problem.

Capacity planning has been an important research topic in Operations Management. However, most of the literature concerning this problem is focused on manufacturing companies (whose characteristics may not fit with many service industries) or very simple services systems (that are often modelled by means of queuing theory).

To the best of our knowledge, there are no formalized tools or procedures to deal with strategic capacity planning in KIOs. Defining the strategic capacity planning problem, identifying the most relevant characteristics and proposing a classification scheme is the first step towards the design of a general solving methodology and the development of specific solving procedures for each case.

The rest of the paper is organized as follows: section 1.2 contains the main findings regarding KIOs and points out the relevant differences between manufacturing companies and KIOs; section 1.3 defines the strategic capacity planning problem in KIOs, introduces the most important characteristics and proposes a classification scheme; finally, section 1.4 contains the conclusions and future research lines.

\subsection{Manufacturing Companies vs Knowledge Intensive Organizations (KIOs)}

As it is said in the introduction, most of papers dealing with strategic capacity planning are focused in manufacturing companies. Service organizations differ from manufacturing industries in a number of ways. One of the most important differences is that most service facilities exist in a restricted local market, so that 
service product cannot be shipped to other markets and it cannot be stocked to meet fluctuations in demand. Second, the economies of scale in service industries are often considerable less than for manufacturing or process industries (Berman et al., 1994).

Also, the service industry is very broad and businesses are different. Knowledge-intensive organizations represent a specific case in the service industry, for which capacity depends on the size and composition of the workforce.

The relevance of the idea of knowledge-intensive firms (KIFs) or, in a general way for any organization, knowledge-intensive organization (KIOs) as a knowledge company has increased in recent years (Alvesson 1993, Kärreman, 2010), even though there is still a lack of consensus on the definition of KIOs (Makani and Marche, 2010).

According to the seminal work of Starbuck (1992) a KIF assumes knowledge as the more important resource, distinct from the labour- and capital-intensive organization. Knowledge differs from other resources in being immaterial and ambiguous (Alvesson, 1993). The principle of organization of knowledge-creating work may differ significantly from the traditional organization of physical work (Choo, 1997).

One KIF's key characteristic is the capacity to solve problems trough creative and innovative solutions (Robertson and Swan, 2003). In this sense, the competitive advantage for KIFs consists of the creation, application and preservation of superior knowledge and expertise within a firm (Starbuck, 1992). Makani and Marche (2010) proposed a typology and analysis of the factors that differentiate KIOs from other organizations. One step further, in order to classify KIOs, these authors identified two dimensions of knowledge intensity: the worker dimension and the organizational/unit dimension. Our research is focused on the knowledgeintensive organizations (KIO), that is, the organizations where knowledge has been regarded as a critical and strategic resource and a key core competence, such as universities, consulting firms or high-tech and engineering firms (Robertson and Swan, 2003).

According to previous ideas, the strategic capacity planning problem in a KIO can be assumed, mainly, to a workforce capacity planning problem in which developing human resource with necessary skills to meet the future demand is a key decision. The problem involves identifying the current and future skill types and numbers of employees required for the organization needs, transferring (from one department or branch to another), hiring and firing (Song and Huang, 2008).

Recently, the problem of managing highly skilled employees has received an increasing research interest as the world is moving from an industrial-based to a more service- and information-based economy (Song and Huang, 2008).

Service sector has an increasing importance in developed economies in recent decades, both in production as in employment. Also, service activities are being incorporated more and more into manufacturing companies. However, the relevance of the services sector in developed countries has not been reflected in the importance given to Operations Management research (a field for which capacity 
design and long-term capacity decisions are one of the main interest topics). As Machuca et al. (2007) pointed out despite the importance of service organizations little attention is still paid to service operations research in the Operations Management field. Regarding KIOs, there are not almost works in Service Operations Management research.

\subsection{Strategic Capacity in KIOs: A Problems' Classification Scheme}

In an organization where knowledge or, in particular, people with specific and difficult to get knowledge, is the main resource (as a university or a consulting company), the strategic capacity planning problem can be basically defined as a strategic workforce (staff, personnel, workers) planning problem.

The problem consists mainly on deciding the number and type of workers to hire (or promote) for each category and to fire, for every period of the planning horizon, which can be from one year up to, for example, ten years (the "long term" can be more or less long depending on the case). Of course, decisions regarding training and transfers (from one department or branch to another) must be also considered.

Economic issues (such as cash management) should be taken into account, and there can be different evaluation criteria such as the profit, the fulfilling of the demand or achieving a staff whose composition (categories, age, etc.) equals an ideal situation.

Even though a general methodology can be developed to deal with this problem, a specific procedure has to be designed ad hoc for each particular case, depending on its characteristics.

\section{Organization Structure}

The organization can be structured by departments or by processes/ projects. The main difference, concerning capacity planning, is that while a worker cannot belong to more than one department, he or she can belong to more than one process/project.

\section{Workforce characteristics}

The workforce is supposed to be organized by categories, and all members of a category are supposed to be able to perform the same set of tasks. If there is crosstraining, a type of task can be performed by more than one category, even though the yield in performing the task can depend on the category. In the opposite case (dedicated categories), a task can be done only by members of one category. 
When a worker is hired or promoted to a category, there may exist a learning period during which the yield performing the task can be low and increasing up to the maximum after a certain number of periods.

Besides the category (which is directly related to the tasks that can be performed and, somehow, with the quality and or speed in performing those tasks), there are other characteristics that can be considered to classify workers: age (or time to the retirement), turnover ratio, etc.

\section{Capacity decisions}

The available capacity depends basically on the size and composition of the staff (and their experience if there is a learning period for the new workers). Of course, a big expansion may lead to the necessity of getting additional space (a new site) so the corresponding costs should be considered. Also, when the KIO is organized by processes or projects, the capacity depend on how the workers are allocated to the different projects.

The capacity decisions may mainly include hiring and firing, promotions and transfers (from one department or one project to another). For the transfers, a training period may be necessary.

Usually it is desirable to consider also some tactical decisions in strategic planning (a system will be better if it is designed taking into account its operation). Regarding capacity, overtime and outsourcing decisions, as well as project or tasks assignments may be included.

\section{Capacity Requirements (Demand)}

The capacity requirements (or demand), or a probabilistic distribution or a set of scenarios of them are supposed to be known. These may depend on uncontrollable factors and on decisions taken also at the strategic level but that are considered to be fixed for the capacity planning, such as the introduction of new products (or services), or the opening to new markets.

The capacity requirements may be considered by category, type of task or project. Also, a minimum, a maximum and a desired or ideal capacity requirement can be defined.

\section{Service Level}

There are many ways of quantifying the service level of a company. For example, it can be forced that the available capacity meets, at least, a certain percentage of the required capacity. Also, it must be considered if demand can be delayed (a consulting can delay the starting of a project; instead, a university cannot delay or stop the course). 


\section{Workforce Costs}

The variable costs to be considered in this problem may include salaries, hiring, firing and training. If from a given number of workers it is necessary to open a new site (or increase the available space) and, possibly, to hire complementary staff (for example, for administrative and IT tasks), the corresponding costs can be assigned to the workforce. In this case, the relation between the number (and type) of workers and the costs will be not linear.

\section{Finance}

Financial planning decisions can be considered together with capacity decisions or not. The expansions or reductions may lead to financial needs that cannot be faced by the organization (in this case, the costs due to getting a loan from a bank should be considered). Also, in high income periods, money can be invested in different ways. Finally, budget constraints can be taken into account

\section{Uncertainty}

Uncertainty may affect many factors such as capacity requirements/demand, economic parameters, workforce turnover, etc. The uncertainty can be faced by solving several deterministic scenarios (in this case, uncertainty is not considering when designing a solving tool) or by means of a stochastic approach.

\section{Planning Horizon}

Strategic Capacity Planning is also known as Long Term Capacity Planning. However, depending on the type of organization (how changing are the conditions), the planning horizon can be more or less long; for example, from one year up to twenty years. The length of the planning horizon may affect the kind of decisions to be included in the planning (for example, to include or not tactical decisions, how to consider financial issues, etc.) and, also, the importance of the uncertainty regarding some parameters.

\section{Evaluation Criteria}

Several strategic capacity planning solutions may be generated or designed, and there are different criteria that can be used to evaluate them and choose the best one. The economical one (for example, the profit) is probably the most used one, but there are other regarding the service level (for example, to get a capacity as close as possible to the desired one) or the composition of the staff (for example, to have a workforce whose composition, in terms of age and category is as close as possible to an ideal one). The different criteria can be considered in a hierarchical way or can be combined into an evaluation function. 


\section{Classification Scheme}

Previous ideas lead to the classification shown in Table 1.1. Even though not all combinations may have sense, the scheme gives rise to a high number of variants.

Table 1.1 Strategic capacity planning in KIOs: a classification scheme

\begin{tabular}{|c|c|c|}
\hline Issue & Characteristic & Options \\
\hline \multirow{2}{*}{ Organization } & \multirow{2}{*}{ Organization structure } & Departments \\
\hline & & Projects \\
\hline \multirow{8}{*}{ Workforce } & \multirow{2}{*}{ Categories } & Dedicated categories \\
\hline & & Cross-training \\
\hline & \multirow{2}{*}{ Age of workers } & $\underline{\text { Relevant }}$ \\
\hline & & Not relevant \\
\hline & \multirow{2}{*}{ Learning effect } & Considered \\
\hline & & Not considered \\
\hline & \multirow{2}{*}{ Workforce turnover } & Considered \\
\hline & & Not considered \\
\hline \multirow{10}{*}{ Capacity decisions } & \multirow{2}{*}{ Hiring \& Firing } & Unlimited \\
\hline & & Limited \\
\hline & \multirow{2}{*}{ Workers promotion } & Unlimited \\
\hline & & Limited \\
\hline & \multirow{2}{*}{ Transfers } & Allowed \\
\hline & & Not allowed \\
\hline & \multirow{2}{*}{ Training } & Included \\
\hline & & Not included \\
\hline & \multirow{2}{*}{ Tactical decisions } & Included (overtime/outsourcing/assignment) \\
\hline & & Not included \\
\hline \multirow{3}{*}{ Demand } & \multirow{3}{*}{ Capacity requirements } & By category \\
\hline & & By type of task \\
\hline & & By process/project \\
\hline \multirow{2}{*}{ Service level } & \multirow{2}{*}{ Actual capacity } & $\%$ of the requirements, without delays \\
\hline & & $\%$ of the requirements, with delays \\
\hline \multirow{4}{*}{ Costs and Finance } & \multirow{2}{*}{ Workforce costs } & Linear \\
\hline & & Not linear \\
\hline & \multirow{2}{*}{ Financial planning } & Included \\
\hline & & Not included \\
\hline \multirow{2}{*}{ Uncertainty } & \multirow{2}{*}{ Stochastic variables } & Considered \\
\hline & & Not considered \\
\hline Planning horizon & Term & Medium (1-5 years) \\
\hline
\end{tabular}




\begin{tabular}{lll}
\hline & Long $(>5$ years $)$ \\
\hline \multirow{3}{*}{ Goal } & Evaluation criteria & Economic \\
\cline { 2 - 2 } & Service level \\
\hline
\end{tabular}

\subsection{Conclusions}

This paper presents a classification scheme for the Strategic Capacity Planning in KIOs, a problem that, to the best of our knowledge, has not been previously dealt with (or, at least, no formalized solving procedures have been proposed). Future research includes designing a general solving methodology and develop solving tools for different variants of the problem.

\subsection{References}

Alvesson M (1993). Organization as a rethoric: knowledge-intensive firms and the struggle with ambiguity. Journal of Management Studies 30:997-1015.

Berman O, Ganz Z, Wagner JM (1994) A stochastic optimization model for planning capacity expansion in a service industry under uncertain demand. Naval Research Logistics 41:545564.

Choo C W (1997). The knowing organization: How Organizations Use Information To Construct Meaning, Create Knowledge, and Make Decisions. Oxford University Press.

Geng N, Jiang Z (2009) A review on strategic capacity planning for the semiconductor manufacturing industry. International Journal of Production Research 47:3639-3655.

Kärreman D (2010). The Power of Knowledge: Learning from 'Learning by KnowledgeIntensive Firm'. Journal of Management Studies 47:1405-1416.

Luss H (1982) Operations research and capacity expansion problems: A survey. Operations Research 30:907-947.

Machuca JAD, González-Zamora MM, Aguilar-Escobar VG (2007) Service operations management research. Journal of Operations Management 25:585-603.

Makani J, Marche S (2010). Towards a typology of knowledge-intensive organizations: determinant factors. Knowledge Management Research \& Practices 8:265-277.

Olhager J, Rudberg M, Wikner J (2001) Long-term capacity management: Linking the perspectives from manufacturing strategy and sales and operations planning 69:215-225.

Paraskevopuolos D, Karakitsos E, Rustem B (1991) Robust capacity planning under uncertainty. Management Science 37:787-800.

Robertson M, Swan J (2003). 'Control- What Control?' Culture and Ambiguity Within a Knowledge Intensive Firm. Journal of Management Studies 40:831-858.

Song H, Huang H-C (2008). A successive convex approximation method for multistage workforce capacity planning problem with turnover. European Journal of Operational Research 188: $29-48$

Starbuck W H (1992). Learning by Knowledge-Intensive Firms. Journal of Management Studies 29:713-740. 